\title{
Magnetic Maps of Indoor Environments for Precise Localization of Legged and Non-legged Locomotion
}

\author{
Martin Frassl $^{1}$, Michael Angermann ${ }^{1}$, Michael Lichtenstern ${ }^{1}$, Patrick Robertson ${ }^{1}$, \\ Brian J. Julian ${ }^{2,3}$, Marek Doniec ${ }^{2}$
}

\begin{abstract}
The magnetic field in indoor environments is rich in features and exceptionally easy to sense. In conjunction with a suitable form of odometry, such as signals produced from inertial sensors or wheel encoders, a map of this field can be used to precisely localize a human or robot in an indoor environment. We show how the use of this field yields significant improvements in terms of localization accuracy for both legged and non-legged locomotion. We suggest various likelihood functions for sequential Monte Carlo localization and evaluate their performance based on magnetic maps of different resolutions. Specifically, we investigate the influence that measurement representation (e.g., intensity-based, vectorbased) and map resolution have on localization accuracy, robustness, and complexity. Compared to other localization approaches (e.g., camera-based, LIDAR-based), there exist far fever privacy concerns when sensing the indoor environment's magnetic field. Furthermore, the required sensors are less costly, compact, and have a lower raw data rate and power consumption. The combination of technical and privacy-related advantages makes the use of the magnetic field a very viable solution to indoor navigation for both humans and robots.
\end{abstract}

\section{INTRODUCTION}

\section{A. Motivation}

Precise localization of humans and robots in indoor environments is an essential component for numerous applications. Existing technical solutions to indoor localization either rely on (i) infrastructure, such as radio beacons, passive or active transponders, guiding wire, and/or optical markings; or (ii) onboard sensors, such as LIDAR (Light Detection and Ranging), monocular cameras, and/or stereo imaging. Recent insights into the structure of the indoor magnetic field have triggered strong interest in the potential use of this field for localization purposes. Firstly, the indoor magnetic field typically shows strong modulation with spectral components over a wide spatial bandwidth (e.g., $\left[1 \mathrm{~m}^{-1}, \ldots, 0.01 \mathrm{~m}^{-1}\right]$, or even wider), suggesting the capability to acquire robust

This work is supported by DLR under Project Dependable Navigation.

This work is sponsored by the Department of the Air Force under Air Force contract number FA8721-05-C-0002. The opinions, interpretations, recommendations, and conclusions are those of the authors and are not necessarily endorsed by the United States Government.

${ }^{1}$ M. Frassl, M. Angermann, M. Lichtenstern and P. Robertson are with the Institute of Communications and Navigation of the German Aerospace Center (DLR), 82234 Wessling, Germany, martin.frass1@dlr.de, michael.angermann@dlr.de, m.lichtenstern@dlr.de and patrick.robertson@dlr.de

${ }^{2}$ Marek Doniec and Brian J. Julian are with the Computer Science and Artificial Intelligence Laboratory, MIT, Cambridge, MA 02139, USA, doniec@mit.edu and bjulian@mit.edu

${ }^{3}$ Brian J. Julian is also with MIT Lincoln Laboratory, 244 Wood Street, Lexington, MA 02420, USA

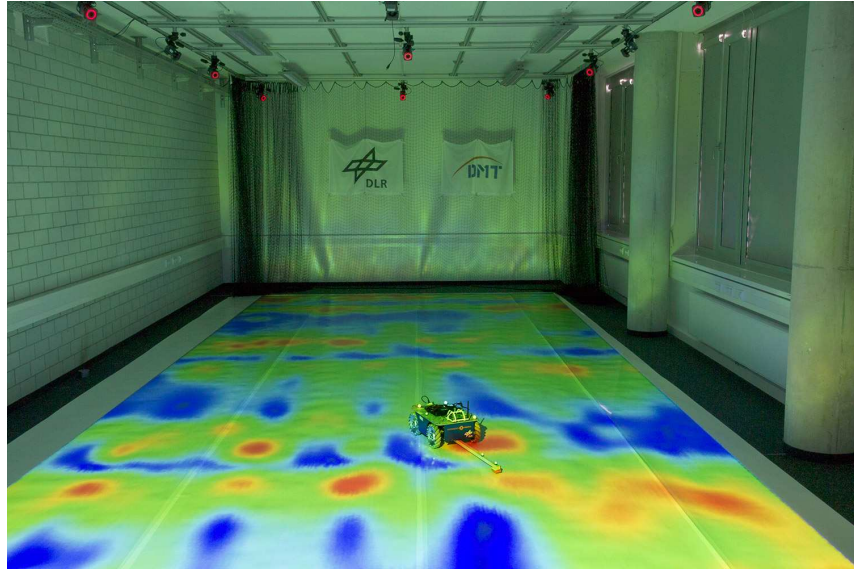

Fig. 1. This photo shows the calibrated projection of the measured magnetic field intensity during a localization run of the robotic platform.

initial location fixes and localize with centimeter-level accuracy. Secondly, the field is stable over long periods of time. Thirdly, suitable sensors to measure the indoor magnetic field are readily available at low cost. Lastly, no additional infrastructure is required.

Depending on the specific application, a further advantage is the relative immunity to environmental conditions such as low lighting or smoke that negatively affects other sensors, particularly cameras or LIDARs. Hence, a combination of magnetic sensing with these other sensors could significantly improve reliability, which is of particular importance in safety and security related applications. Furthermore, we consider magnetic sensors to be less intrusive upon privacy than cameras or LIDARs which obtain imagery or geometry. Privacy concerns may be a severe hindrance in consumer oriented applications. In this light use of the magnetic field may be a more viable alternative.

The magnetic field also appears to be stable over long periods of time due to the static nature of building materials that affects this field, such as reinforced concrete or metal door frames. Distortions can appear due to moving ferromagnetic structures, e.g., elevators, escalators, or furniture. Lastly, the magnetic field gives location information in a very compact vector representation that is directly observable using readily available magnetic sensors. Consequently, the computational effort to extract the relevant information is relatively low, which yields desirable properties in terms of power consumption by personal devices or robotic platforms. 


\section{B. Magnetic Field Characteristics}

The characteristics of the undisturbed earth magnetic field depend on both the location and time of the observation. The magnitude of the temporal change is relatively small with daily fluctuations between $10 \mathrm{nT}$ to $30 \mathrm{nT}$, which is less than $0.1 \%$ of the average magnitude of $48.19 \mu \mathrm{T}$ seen in our geographic region [1]. The spatial change, however, has a significant influence on the direction and magnitude of the magnetic field. The magnetic field vector roughly points in the direction of geographic north, but is more accurately represented by its declination and inclination. The declination describes the horizontal deviation of the direction from the geographic north, while the inclination describes the vertical component of the field. Approximate values of $145^{\prime}$ declination and $64^{\circ}$ inclination are reported at the location of our lab facilities $\left(48.08^{\circ} \mathrm{N}, 11.28^{\circ} \mathrm{E}\right)$ [1]. Inclination and magnitude of the magnetic field vector are lowest around the equator and roughly increase with increasing latitude.

The ubiquitous magnetic field is further disturbed by manmade structures. In particularly, the magnetic field is influenced in proximity of ferromagnetic materials used in the construction of buildings - a comprehensive analysis of this property can be found in [2]. For example, Fig. 2 shows the intensity of the magnetic field at ground level within our motion capture laboratory. This intensity varies varies between $0 \mu \mathrm{T}$ and $120 \mu \mathrm{T}$, which is a range that is factors of 2.5 and $10^{4}$ compared to the intensities of the undisturbed magnetic field and its daily fluctuation, respectively.

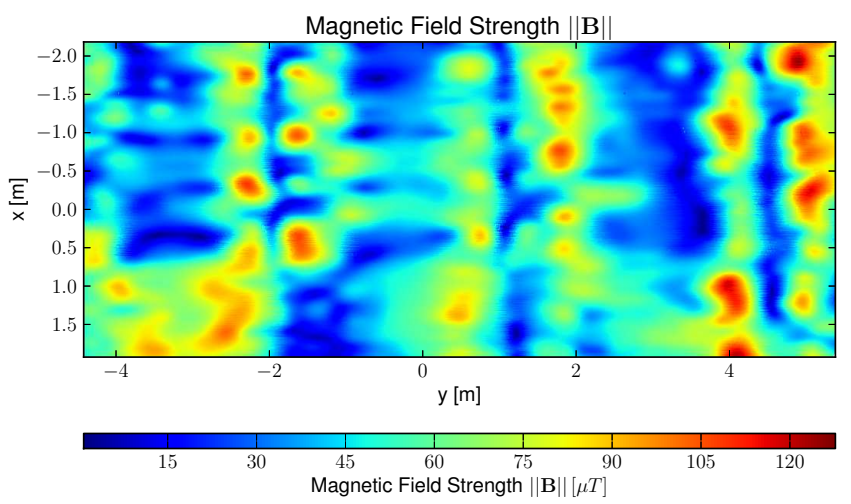

Fig. 2. Map of the magnetic field intensity at ground level within DLR's motion capture laboratory with a spatial resolution of $1 \mathrm{~cm}$.

Fig. 3 shows the horizontal component of the magnetic field from Fig. 2. The color of the field lines is set according to the this component's intensity, which is much less intensive than the corresponding full vectors. Magnetic north points to $\approx 213^{\circ}$ as indicated by the compass in the upper right corner. Fig. 4 shows a histogram representation of the same field, sampled on a $1 \mathrm{~cm}$ grid. It is clearly seen that the field is oriented along the main axes of the building, which align to the plots $\mathrm{x}$ - and $\mathrm{y}$-axes.

\section{Related Work}

The most substantial body of related work exists in the areas of robot localization, mapping, and simultaneous localization and mapping (SLAM). These works employ ground,

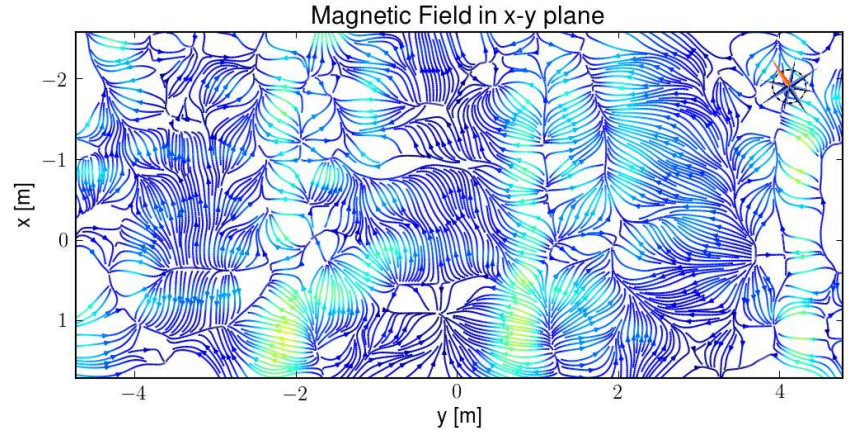

Fig. 3. The horizontal component $\left(B_{x}, B_{y}\right)$ of the magnetic field at ground level within DLR's motion capture laboratory. The color scale is set according to the one in Fig. 2.

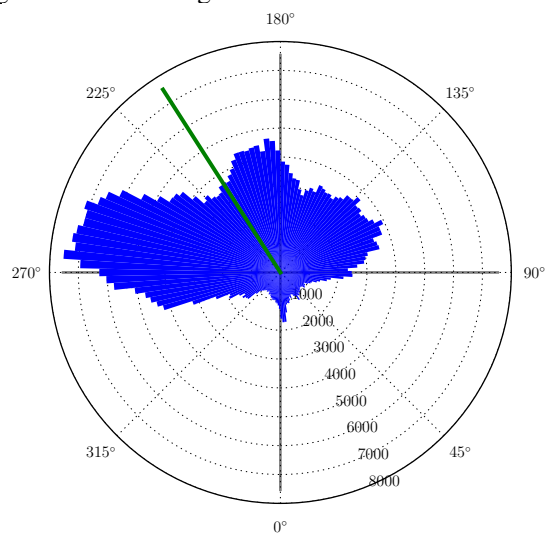

Fig. 4. Histogram of the directions of the horizontal component $\left(B_{x}, B_{y}\right)$ of the magnetic field at ground level within DLR's motion capture laboratory, sampled on a $1 \mathrm{~cm}$ grid. The green line denotes the magnetic north, black lines the orientation of the main axes of the surrounding building.

air, and underwater robots using various sensors and types of locomotion. Drawing from experiments and theoretical foundations laid in robotics, there are growing efforts to achieve accurate and robust localization for human pedestrians [3]. In addition, the groundbreaking work of Smith et al. [4], Leonard and Durrant-Whyte [5], Thrun et al. [6], and others have resulting in a strong understanding of localization and SLAM solutions based on active sensors such as sonars, LIDARs, and optical cameras. These efforts have resulted in fieldable technologies that serve in numerous industrial, exploration [7], and transportation [8] applications.

Due to their ability to perform with cost efficient monocular camera sensors, the works by Davison et al. [9] and Klein et al. [10] are important steps towards economic feasibility of mass market applications for indoor localization. For continuous pedestrian localization, many approaches draw on some kind of human odometry, such as the pioneering work by Foxlin using foot mounted inertial sensors [11]. To constrain the inevitable odometry drift error process, one can draw on knowledge of constraints such as walls, which can be inferred using Simultaneous Localization and Mapping without additional exteroceptive sensors [12], [3]. However, for special application, such as rapid response missions by emergency personnel, the requirement in terms of accuracy, robustness and speed of convergence strongly mandate the use of exteroceptive sensors. Fallon et al. designed a system that fuses information from RGB-D cameras, LIDAR, inertial 
sensors, and barometric sensors [13]. This approach is able to robustly localized in realtime for complex 6-DOF motion and challenging trajectories on, for example, stairs and elevators.

Both academia and industry have recently shown great interest in using the indoor magnetic field for localization. This interest is motivated by recent findings on the informativeness of the magnetic field and the extremely low cost of available sensors. Employing an iRobot Create mobile robot platform equipped with a 3-axis magnetometer, Vallivaara et al. were the first to generate geometrically consistent maps of the magnetic field using SLAM [14]. Their work provides significant inspiration for us, and we wish to extend their results by investigating the magnetic field as close to the ground as possible, where we expect a more temporally stable and spatially informative field.

Le Grand and Thrun describe the use of magnetic sensors in a smartphone to build a map of the magnetic field, which could enable very economical mass market applications [15]. The authors use a similar algorithm as described in this work, but measure the magnetic field at a higher height and do not use odometry information. Additionally, no high precision ground truth system was used for mapping or localization reference. [16] and [17] propose leader-follower systems to navigate groups of robots following a magnetic trajectory measured by a leading robot. In [16] the authors use a 1D map and a Kalman Filter for localization in a corridor. In [18] the authors describe the historicals development and current efforts to use large scale (natural) magnetic anomalies for aircraft positioning. Among the most challenging application of magnetic localization has been proposed and successfully demonstrated by Moore et al. for perching aerial robots on power lines [19]. In our own previous work, we have analyzed the properties of the indoor magnetic fields with respect to their potential use for localization of robots and pedestrians [2]. These and other recent findings [20] by Kim et al. have strongly motivated our research.

The idea of using foot mounted sensors for legged localization is not limited to humans. In fact, there is much potential in using biped [21], [22], [23] or quadruped robots [24], [25] to autonomously map the magnetic field of a 3D volume space just above floor level. The resulting abundance of information characterizing indoor environments will not only serve as "infrastructure" for indoor pedestrian localization, but also in progressing the state of motion planning and control for legged robots [26]. Such databases can also serve as replacements for more expensive localization methods in the robotics community, both for basic research (e.g., gate analysis [27]) and for technology demonstrations (e.g., Robocup [28]).

\section{Problem Statement}

Consider the problem of localizing a subject in an indoor environment, where the subject's locomotion can be legbased (e.g., humans, biped robots, quadruped robots) or nonlegged-based (e.g., wheelchairs, Ackermann vehicles). We focus on the two-dimensional localization problem, meaning that the subject's pose $\mathbf{P}_{k}$ at time step $k \in \mathbb{Z}_{\geq 0}$ is a random variable ${ }^{1}$ that takes values from $\mathbb{R}^{2} \times \mathbb{S}$, i.e., the twodimensional Euclidean space with corresponding rotation. For multiple pose hypotheses, we are interested in determining the hypothesis associated with the highest posterior probability $p\left(\mathbf{P}_{k} \mid\left\{\mathbf{Z}^{U} \mathbf{Z}^{B}\right\}_{1: k}\right)$, where the measurement sequence $\left\{\mathbf{Z}^{U} \mathbf{Z}^{B}\right\}_{1: k}$ is composed of odometry measurements $\mathbf{Z}_{1: k}^{U}$ and magnetic field measurements $\mathbf{Z}_{1: k}^{B}$.

\section{A. Indoor Magnetic Environment}

Assuming a time-stationary indoor magnetic environment, we model the a priori magnetic map $B: \mathbb{R}^{2} \times \mathbb{S} \rightarrow \mathbb{R}^{3}$ as a vector field. More specifically, given a pose $\mathbf{P}_{k}$ within the magnetic map's domain, the map projects this pose to a three dimensional vector $B\left(\mathbf{P}_{k}\right)$. Note that for any $b \in \mathbb{R}$, the magnetic field vector $(0,0, b)$ is parallel to the local gravity vector.

\section{B. Odometry and Magnetic Field Measurements}

In this paper we rely on sources of odometry measurements and co-located magnetic field measurements. Typically, the odometry is derived from inertial sensors for legged locomotion and rotary encoders for wheeled locomotion. Inertial sensors, in addition to providing odometry that is differential, are also informative with respect to the subject's absolute attitude, as discussed in Section III-A.2. For simplicity of notation, let $\Delta x_{k}^{U} \in \mathbb{R}^{2}$ and $\Delta \Theta_{k}^{U} \in \mathbb{S}$ denote the measured change in translational and rotational position, respectively, derived from the odometry measurement $\mathbf{Z}_{k}^{U}$, for all times $k$.

By the term co-located, we mean that the magnetic field measurement $\mathbf{Z}_{k}^{B}$ is taken at the pose $\mathbf{P}_{k}$, for all times $k$. This measurement is of vector form $\left(\mathbf{Z}_{k}^{B_{x}}, \mathbf{Z}_{k}^{B_{y}}, \mathbf{Z}_{k}^{B_{z}}\right) \in \mathbb{R}^{3}$, and is implemented in various likelihood functions, as discussed in Section III-A. We make the assumption that the sensor is always close enough to the ground as to be subjected to the ground-level magnetic field.

\section{Algorithms}

We are interested in legged and non-legged locomotion for which some form of odometry is available. Since this odometry is affected by noise but is still informative, it lends itself to serve in the prediction step of a Bayesian filter. Following the prediction step, the resulting hypotheses are evaluated in the update step of the filter. This evaluation considers how well the actual measurements from the magnetic sensor match the magnetic map's values at the subject's predicted pose. Since the likelihood functions and probability distributions present in this Bayesian filter are strongly multi-modal, we have chosen to use a particle filter (also known as a sequential Monte Carlo filter) with systematic resampling [29].

We assume the odometry is reasonably accurate from one time step to the next, and we employ a proposal function that randomly draws the next pose of a particle given its previous pose, its error states of the odometry, and the odometry measurement itself. The update step of the Bayesian filter

\footnotetext{
${ }^{1}$ We denote all random variables as bold faced capital letters.
} 
then employs the measurement likelihood computed at the particle's pose given the magnetic field map. Specific to legged localization using inertial sensors, we believe that this proposal function is justified because, conditioned on knowledge of the odometry's error state such as angular drift, prediction of the displacement vector between (legged) steps is typically in the order of a centimeter [30]. This magnitude is of the same order as the expected discrimination of the magnetic field likelihood function per measurement [2].

\section{A. Likelihood Functions}

Consider a population of $N_{p}$ particles indexed by $i \in\left\{1, \ldots, N_{p}\right\}$. Each particle has a corresponding pose $\left(x^{[i]}, \Theta^{[i]}\right) \in \mathbb{R}^{2} \times \mathbb{S}$, odometry drift rate $\tau^{[i]}$, and weight $w^{[i]}$, where the pose is projected to a three dimensional magnetic field vector $B^{[i]}:=B\left(\mathbf{x}^{[i]}, \Theta^{[i]}\right)=\left(B_{x}^{[i]}, B_{y}^{[i]}, B_{z}^{[i]}\right)$ by the magnetic map. We now discuss three likelihood function variants, each of which uses the magnetic field measurement $\mathbf{Z}_{k}^{B}$ to update the weights of the particles at time $k$. Note that this update step of the particle filter is invoked only for particles that reside within the mapped area, i.e., in the domain of $B$. The proposal, update, and resampling steps of the filter are summarized in Algorithm 1, where the given values are specific to our legged locomotion application.

1) Magnetic Field Intensity (1D): The first likelihood function variant employs the difference between the magnitudes of the magnetic field vectors $\mathbf{Z}_{k}^{B}$ and $B^{[i]}$. More specifically, the difference between the Euclidean norms (i.e., $\left\|\mathbf{Z}_{k}^{B}\right\|_{2}-\left\|B^{[i]}\right\|_{2}$ ) is used to generate a measurement likelihood from a Gaussian mixture model (GMM). This GMM consists of two fixed variance Gaussian distributions, the first being a narrow distribution and the other a wide one. The measurement likelihood is bounded from below by a fixed value before updating the weight of a particle.

2) Vertical and Horizontal Magnetic Field Components (2D): The second likelihood function variant employs the difference between the vertical magnetic field components $\mathbf{Z}_{k}^{B_{z}}$ and $B_{z}^{[i]}$, as well as the difference between the magnitudes of the horizontal magnetic field components $\mathbf{Z}_{k}^{B_{h}}:=$ $\left(\mathbf{Z}_{k}^{B_{x}}, \mathbf{Z}_{k}^{B_{y}}\right)$ and $B_{h}^{[i]}:=\left(B_{x}^{[i]}, B_{y}^{[i]}\right)$. This variant exploits the strength and stability of the local gravity vector, since the tilt angle of the subject can be determined with good accuracy if the source of odometry measurements comes from an inertial-based sensor. Once the tilt angle is known, the vertical difference $\mathbf{Z}_{k}^{B_{z}}-B_{z}^{[i]}$ and the horizontal difference $\left\|\mathbf{Z}_{k}^{B_{h}}\right\|_{2}-\left\|B_{h}^{[i]}\right\|_{2}$ can be calculated to generate a likelihood from the same Gaussian distribution of fixed variance. The (bounded) product of these likelihoods forms the measurement likelihood used to update the particle weight.

3) Magnetic Field Vector (3D): The third likelihood function variant employs element-wise differences between magnetic field vectors $\mathbf{Z}_{k}^{B}$ and $B^{[i]}$. More specifically, the elements of the vector $\mathbf{Z}_{k}^{B}-B^{[i]}$ are each used to generate a likelihood from the same Gaussian distribution of fixed variance. The product of these likelihoods is again bounded from below by a fixed value to form a measurement likelihood used to update the weight of the particle.

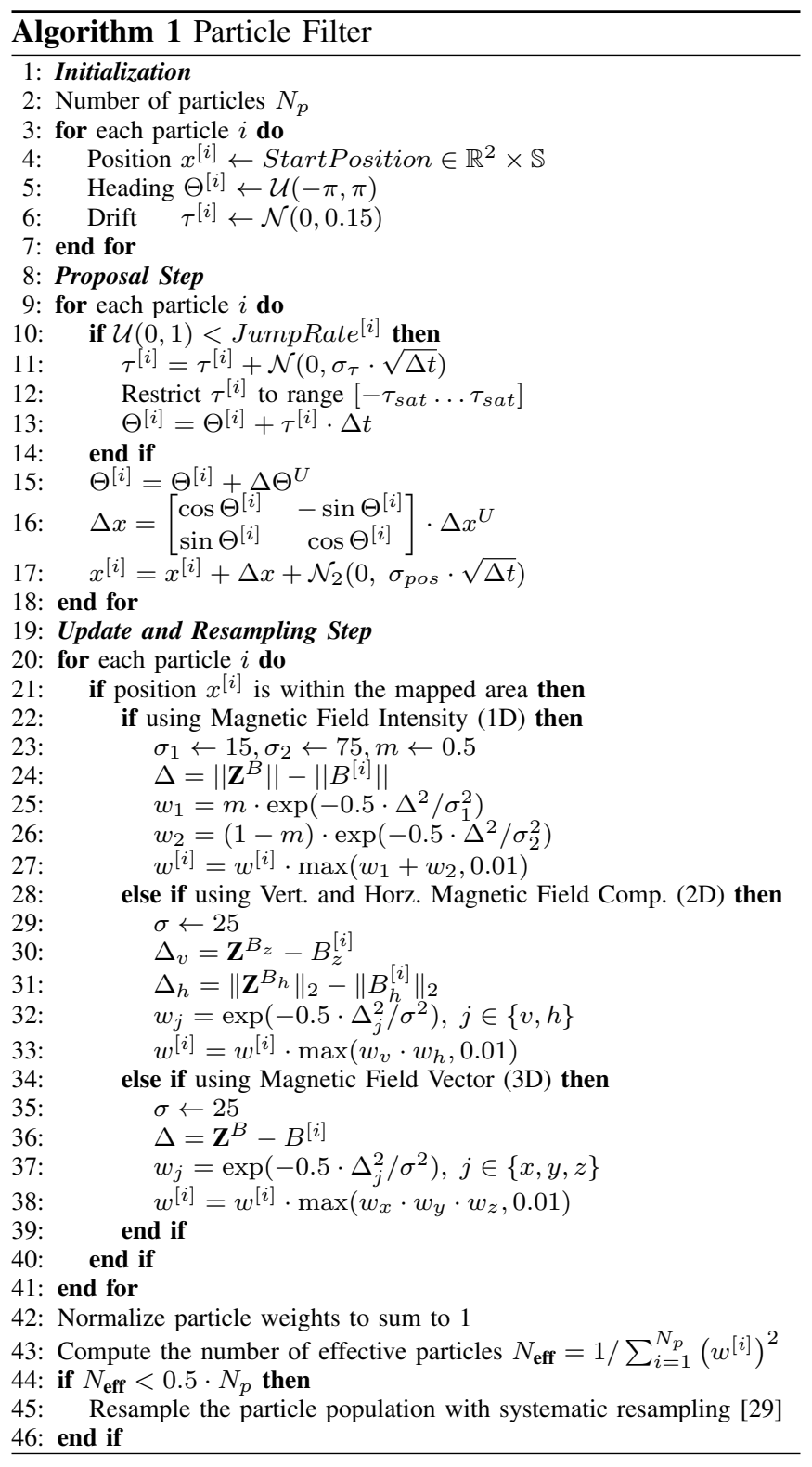

\section{EXPERIMENTAL SETUP}

\section{A. Ground Truth Tracking}

We employed a commercial motion capture systems (Vicon) to provide accurate pose information during the mapping phase as well as ground truth information the localization experiments. For our particular setup consisting of 16 infrared sensitive cameras and infrared strobes, the system yields sub-centimeter pose accuracy at a rate of $100 \mathrm{~Hz}$.

\section{B. Sensors}

The magnetic field sensor module used in the reported experiments use three orthogonal thin film magneto-resistive sensor units. This triad of sensors is part of commercial integrated sensor packages (Xsens MTx and its wireless variant Xsens MTw). In addition to a magnetic sensor triad, these packages contain accelerometer and gyrometer triads. For the MTx package, the sensor communicates via USB using 
a proprietary protocol for configuration and data logging; the MTw uses a proprietary wireless communications link operating in the $2.4 \mathrm{GHz}$ ISM band. For the experiments, both the odometry measurements $\mathbf{Z}_{k}^{U}$ and magnetic field measurements $\mathbf{Z}_{k}^{B}$ are received at $100 \mathrm{~Hz}$.

We used these sensors for the robotic magnetic mapping, the non-legged localization, and the pedestrian localization. For the robotic mapping, we mount the sensor on a wooden beam that extended $0.75 \mathrm{~m}$ from the center of the robot. The purpose of this beam is to separate the sensor from the robot's ferromagnetic components (e.g., bearings, steel screws) and electromagnetic field generating devices (e.g., motors, motor drivers). We note that the distance of $0.75 \mathrm{~m}$ was selected due to its sufficient separation yet reasonable length with respect to the difficulty of positioning a long "lever arm."

\section{Robotic Platform}

In this work, we use an omnidirectional robot to perform mapping as well as to analyze localization performance for wheeled platforms. The robot is a modified version of the commercially available Slider platform by Commonplace Robotics, see Fig. 5. The platform's chassis made of aluminum sheet metal, and its overall dimensions are $450 \mathrm{~mm} \times 300 \mathrm{~mm} \times 170 \mathrm{~mm}$ (length $\times$ width $\times$ height). The drive system consists of two motor drivers with two channels, four gearmotors with magnetic encoders, and four Mecanum wheels of $150 \mathrm{~mm}$ diameter.

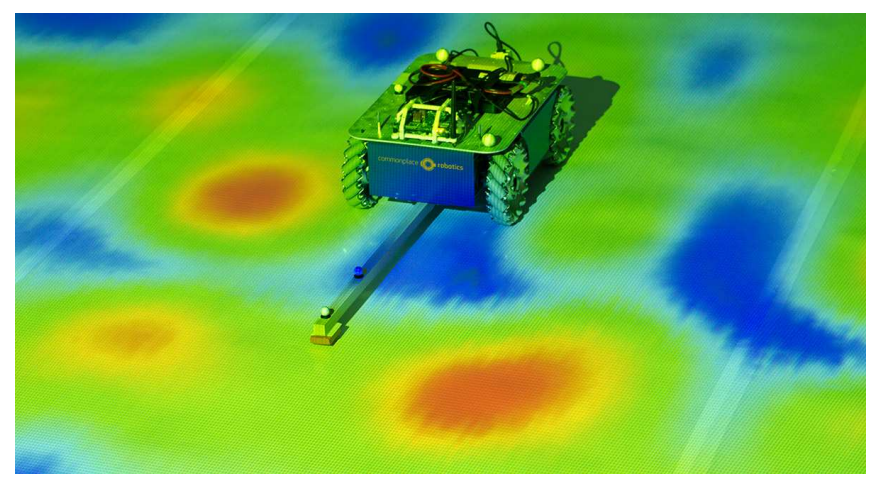

Fig. 5. Robotic platform with sensor arm in a calibrated projection of the magnetic field intensity.

The platform is fully holonomic and accepts forward, lateral, and rotational velocity control inputs. The differential measurements $\left(\Delta x_{k}^{U}, \Delta \Theta_{k}^{U}\right)$ are derived from the odometry measurements $\mathbf{Z}_{k}^{U}$ received from the four wheel encoders at a rate of $50 \mathrm{~Hz}$.

\section{Foot-mounted Sensor Platform}

For the legged locomotion experiments, we rigidly mounted the sensor packages described in Section IV-B onto a human's shoe, see Fig. 6. The odometry measurements $\mathbf{Z}_{k}^{U}$ from the inertial sensors are processed with a ZUPT/ZARU/MARU-aided Unscented Kalman filter in accordance to [31] to derive the differential measurements $\left(\Delta x_{k}^{U}, \Delta \Theta_{k}^{U}\right)$ of the foot. We not only use the odometry measurements during the stance phase, but also during the

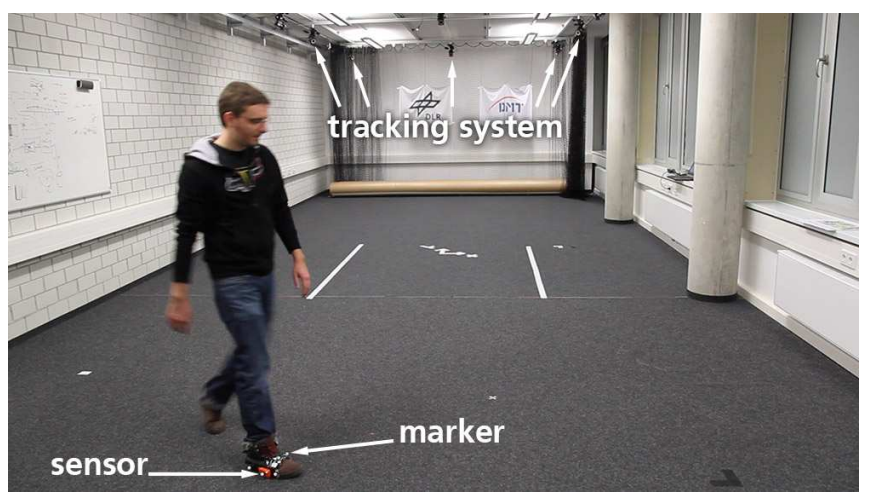

Fig. 6. A pedestrian wearing a shoe equipped with a MTw sensor and reflective IR markers.

stride phase of the step movement cycle. Additionally, we equipped the shoe with reflective markers in order to obtain ground truth trajectory data from the motion tracking system.

\section{E. System Setup and Software Framework}

We extensively used the Robot Operating System (ROS) Framework [32] to acquire various sources of data (ground truth poses from motion tracking, magnetic measurement data, wheel odometry) and to control our wheeled robot. We implemented our particle filter using the Python programming language. Lastly, we used NumPy, Matplotlib [33], and OpenCV to compute the homography of the magnetic field and project it in realtime on the floor of our motion capture laboratory, see Fig. 1.

\section{EXPERIMENTS AND RESULTS}

\section{A. Magnetic Field Mapping}

In preparation for our localization experiments, we created two separate maps of the magnetic field in our motion capture laboratory. The first map was created in December 2012 by manually sweeping the sensor at ground-level using a tackle, which resulted in a map grid size of $10 \mathrm{~cm}$. The second map was created in March 2013 by automating our ground robot (Fig. 5), which resulted in a higher resolution map of $1 \mathrm{~cm}$ grid size. Both mapping methods used the motion capture system discussed in Section IV-A to determine the locations of the magnetic field measurements, and the resulting ground-level maps of the three dimensional magnetic field were approximately $40 \mathrm{~m}^{2}$. The similarities between these two maps constructed four months apart suggest that the magnetic field within our motion capture laboratory is temporally stable over long periods of time.

\section{B. Non-legged Robot Localization}

We have carried out a number of experiments to investigate the localization performance for non-legged locomotion. More specifically, we aimed to localize the holonomic wheeled platform described in Section IV-C. In a first experiment, we manually commanded the robot by setting its forward, lateral, and rotational velocities with a computer input device. Fig. 7 shows in green the ground truth trajectory measured by the motion capture system, while the odometry is shown in red. 


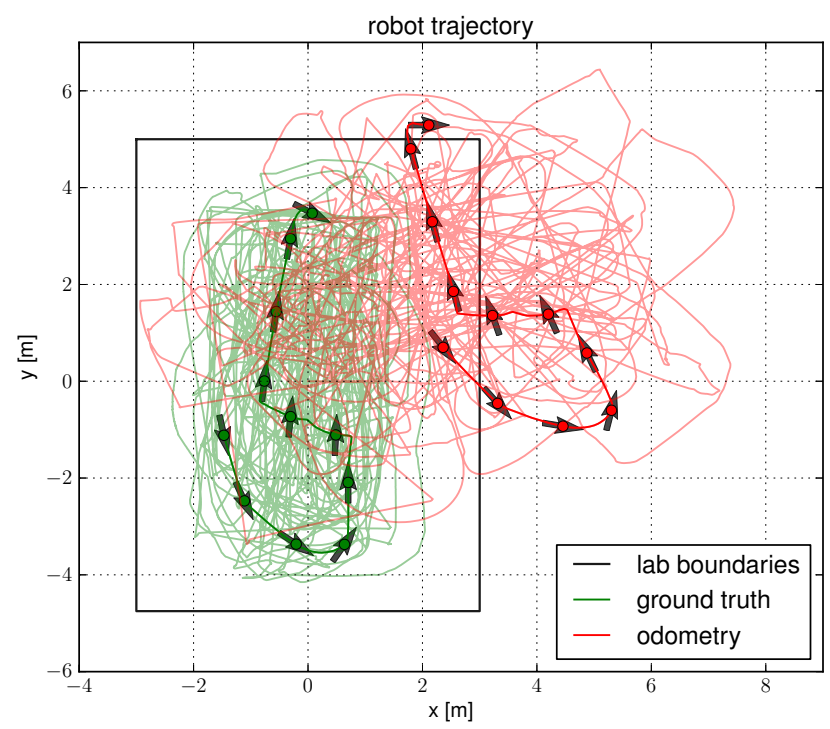

Fig. 7. Ground truth and odometry trajectories for a robot run. The background lines show the complete trajectories while the bold lines show an extract of approximately one minute. The arrows show intermediate position and heading samples of the robot.
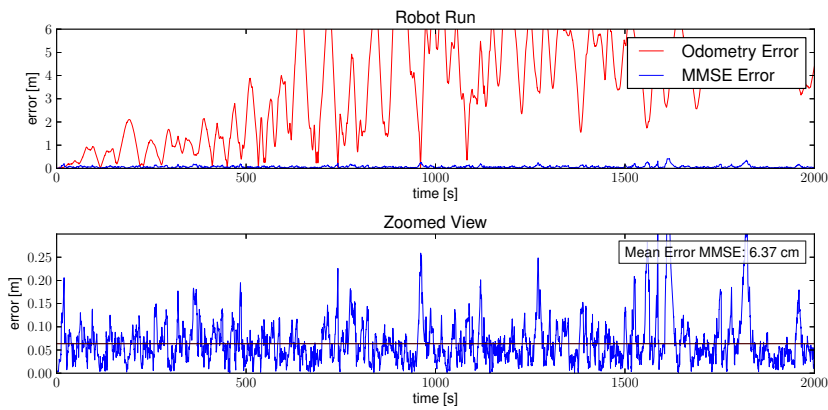

Fig. 8. MMSE Error values for a robot location run of 2000 seconds using the intensity-based likelihood function.

We then processed this odometry using the particle filter, which employed the magnetic map $B$ and the magnetic measurements $\mathbf{Z}_{k}^{B}$ in its update step. Fig. 8 shows the results for a 2000 seconds run with given initial position and heading using the intensity based likelihood function described in Section III-A.1. The mean error over the whole run is 6.4 $\mathrm{cm}$. Note that a known starting position and rotation reduce the error in the initial phase of the run, but the filter also converged without knowing these values.

\section{Legged Pedestrian Localization}

Two different human subjects each performed walks within our laboratory while the inertial measurements $\mathbf{Z}_{k}^{U}$ and magnetic measurements $\mathbf{Z}_{k}^{B}$ were recorded at a rate of $100 \mathrm{~Hz}$, see Fig. 9 for an example trajectory. The subjects began each walk in the center of the tracking area and constrained their motion to within the magnetic map's domain. One of the walks included periods of running and fast turns in order to stress the underlying odometry. Using the motion capture system, we recorded ground truth data at a rate of $100 \mathrm{~Hz}$. Following the same approach as in the non-legged locomotion case from Section V-B, we processed

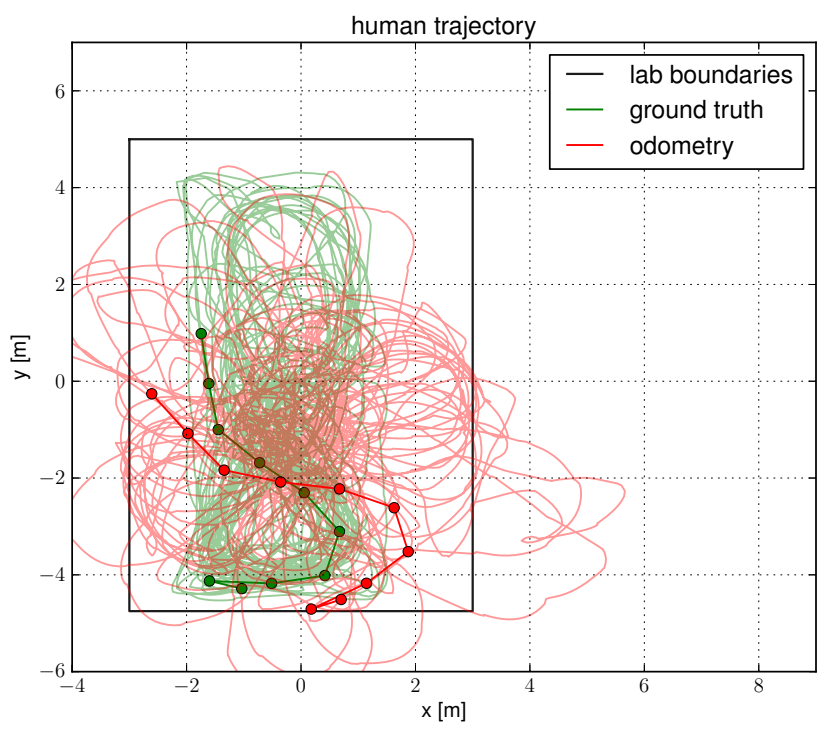

Fig. 9. Trajectories of a pedestrian localization walk.

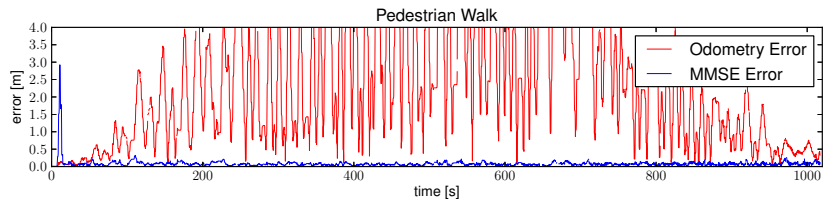

Zoomed View

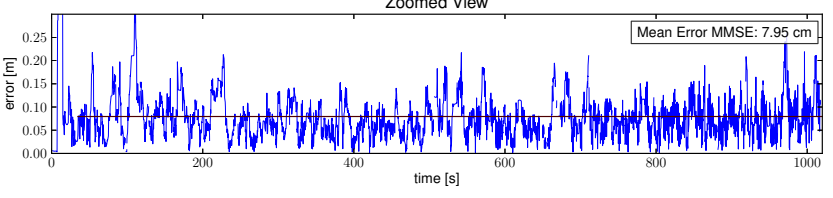

Fig. 10. MMSE Error values for a pedestrian locomotion walk of 1020 seconds using the intensity-based likelihood function.

the data using the particle filter. The resulting error for the intensity-based likelihood function is plotted in Fig. 10. In the pedestrian case, the initial position was given, but the initial heading was unknown and hence estimated by the filter. We observed a stable position error during all walks. For the walks shown in Fig. 8, we used the magnetic map with $10 \mathrm{~cm}$ resolution and the particle filter with $N_{P}=2000$ particles, which resulted in a mean error of $7.95 \mathrm{~cm}$.

\section{Influence of Grid Size}

The magnetic map is stored in a grid representation, for which the resolution has an influence on localization performance. We performed localization on several pedestrian walks using various grid resolutions, see Fig. 11 for an error timeline of walks with two different map resolutions and Fig. 12 for a comparison of the mean error for different map resolutions. Lower resolution maps were generated by sub-sampling from the high resolution map. The poor performance of the $1 \mathrm{~cm}$ map stems from calibration errors between the sensor and motion capture system during the mapping phase, which will be corrected for future experiments. Surprisingly, lower resolution maps still provide sufficient information for indoor localization. For example, the $20 \mathrm{~cm}$ magnetic map resulted in a mean error of less than 


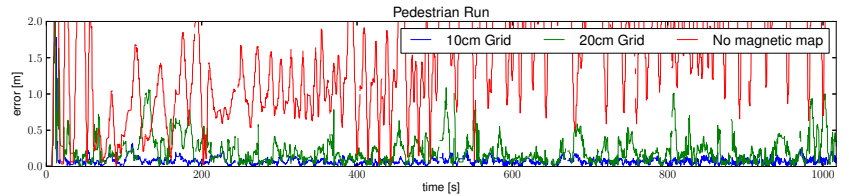

Fig. 11. MMSE Error values for a pedestrian locomotion walks comparing different map resolutions.

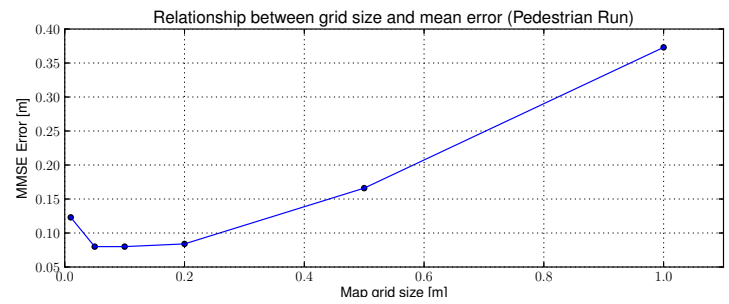

Fig. 12. This plot shows the relationship between grid size of the magnetic map and the resulting MMSE error of the particle filter localization algorithm.

$9 \mathrm{~cm}$, which is essentially equivalent to the $10 \mathrm{~cm}$ map. The red line in Fig. 11 shows the mean error of a walk without the use of any magnetic measurements.

\section{E. Comparison of Likelihood Function Variants}

We employed the particle filter for different representations of the magnetic field map and different variants for the likelihood function as described in Section III-A. Fig. 13 shows a comparison of the mean MMSE for the three likelihood functions. Fig. 14 shows two enlarged temporal segments from Fig. 13, specifically the initialization phase and a period at around 685 seconds with a temporary increase in the error for the intensity based estimator.

TABLE I

COMPARISON OF MEAN ERRORS FOR DIFFERENT MAP GRID RESOLUTIONS AND LIKELIHOOD FUNCTIONS

\begin{tabular}{|l|c|c|c|}
\hline & Intensity & Hor/Ver Components & Full Vector \\
\hline $10 \mathrm{~cm}$ & $9.00 \mathrm{~cm}$ & $7.72 \mathrm{~cm}$ & $7.31 \mathrm{~cm}$ \\
$20 \mathrm{~cm}$ & $9.41 \mathrm{~cm}$ & $8.41 \mathrm{~cm}$ & $7.77 \mathrm{~cm}$ \\
\hline
\end{tabular}

While the mean overall errors for the different likelihood functions do not vary significantly (see Table I), we do observe differences in their robustness and their time for filter convergence. In particular, we notice a degradation when using the intensity based likelihood function, especially for the coarser map. This might be because the intensity map looses trajectory discrimination power for coarser maps in contrast to the richer vector maps.

\section{F. Dataset}

We made our datasets available for download to allow other researchers to work with our high precision data. These datasets include magnetic maps, ground truth trajectories, IMU data, magnetic raw data, and filtered odometry. Please visit http://www.kn-s.dlr.de/indoornav/refdataset.html for more information.

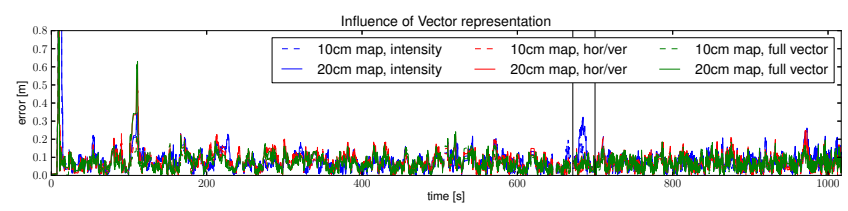

Fig. 13. MMSE values during six pedestrian locomotion walks for three different likelihood functions and two different map resolutions.
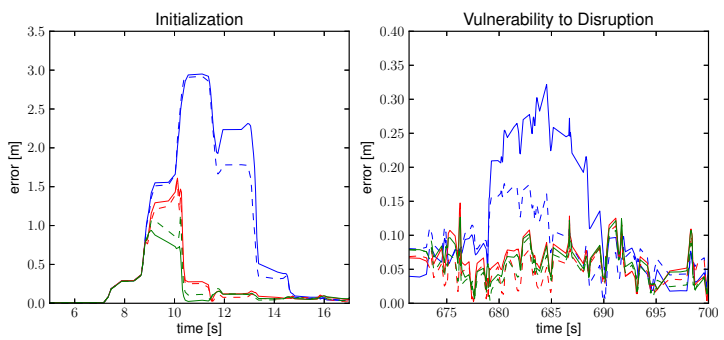

Fig. 14. Zoom into two interesting segments of Fig. 13. On the left side the initialization phase of the particle filter and on the right side a period with a temporary increasing error of the MMSE is shown. In both cases the vectorized representation leads to a smaller error.

\section{Conclusions And Outlook}

In this paper, we investigate the viability of localizing humans and robots in an indoor environment given sources of odometry and co-located magnetic field measurements. This approach uses a prior map of the local magnetic field just above the floor, which was systematically constructed for our experiments using a motion capture system and a three-axis magnetic sensor. The same motion capture system was also used to obtain ground truth poses for evaluating the performance of our localization approach. We showed that previous discussions and hypotheses [2] concerning achievable accuracies are indeed realistic. We also showed that the representations of the magnetic map and the likelihood functions influence localization performance, although even simple representations yielded acceptable performance. For example, a average error of $9 \mathrm{~cm}$ was achieved using the intensities of the magnetic field measurements on a magnetic map of $10 \mathrm{~cm}$ grid resolution. We believe that our experiments were successful for two main reasons. Firstly, we used realistic sources of odometry for pedestrians wearing a foot mounted sensor [11] and for non-legged robots using wheel encoders. Secondly, we took a Bayesian approach to extract salient information of the magnetic field close to the ground, which is shown to be rich in local variation. We see two particularly rewarding challenges for future work: (i) realtime localization for pedestrians with smartphones in their hands or pockets and (ii) Simultaneous Localization and Mapping (SLAM) for both robots and humans. From a practical perspective, we wish to shed the necessity of the motion capture system during the mapping phase.

Future work should also further investigate the relative merit of using just the intensity or one of the two vector representations of the magnetic field. In particular, the relative sensitivity of the field to vertical deviations in the sensor height from the mapped level could be a subject for further studies (this is relevant for the case of legged motion where the sensor is not always at ground level during 
the entire stride). Data sets in different environments would also provide further evidence whether the intensity map is sufficient, especially when more events have been captured where the estimated location suffers from error bursts.

We see a rich set of application scenarios for our proposed approach of robot-based mapping and subsequent localization for legged and non-legged locomotion. The mapping phase could be assigned to floor cleaning or specialized ground robots, and the resulting maps could then be shared with other robotic or human users of the building. We believe a "mild" form of SLAM is sufficient to keep maps current by incorporating newly accessible regions or changes in the field due to moved, added or removed steel structures. One should note that a map of the magnetic field reveals potentially less sensitive information about the indoor environment than images; this property is relevant when addressing privacy concerns. Furthermore, drawing on an additional source of pose information from known magnetic field disturbances should improve the continuity, integrity, and accuracy of systems that use other maps and sensors.

\section{REFERENCES}

[1] LMU Geophysics, "Monthly magnetograms," http://www.geophysik. uni-muenchen.de/observatory/geomagnetism/monthly-magnetograms, last accessed: August 7th, 2013.

[2] M. Angermann, M. Frassl, M. Doniec, B. Julian, and P. Robertson, "Characterization of the indoor magnetic field for applications in localization and mapping," in Indoor Positioning and Indoor Navigation (IPIN), 2012 International Conference on, nov. 2012.

[3] M. Angermann and P. Robertson, "FootSLAM: Pedestrian simultaneous localization and mapping without exteroceptive sensors hitchhiking on human perception and cognition," Proceedings of the IEEE, vol. 100, no. Special Centennial Issue, pp. 1840-1848, 13.

[4] R. Smith, M. Self, and P. Cheeseman, "Autonomous robot vehicles." New York, NY, USA: Springer-Verlag New York, Inc., 1990, ch. Estimating uncertain spatial relationships in robotics, pp. 167-193.

[5] J. Leonard and H. Durrant-Whyte, "Simultaneous map building and localization for an autonomous mobile robot," in Intelligent Robots and Systems '91. 'Intelligence for Mechanical Systems, Proceedings IROS '91. IEEE/RSJ International Workshop on, Nov 1991.

[6] M. Montemerlo, S. Thrun, D. Koller, and B. Wegbreit, "Fastslam: a factored solution to the simultaneous localization and mapping problem," in Eighteenth national conference on Artificial intelligence. Menlo Park, CA, USA: American Association for Artificial Intelligence, 2002, pp. 593-598.

[7] R. M. Eustice, H. Singh, J. J. Leonard, and M. R. Walter, "Visually mapping the RMS Titanic: Conservative covariance estimates for SLAM information filters," Int. J. Rob. Res., vol. 25, no. 12, pp. 1223 1242, Dec. 2006.

[8] C. Urmson and W. Whittaker, "Self-driving cars and the urban challenge," Intelligent Systems, IEEE, vol. 23, no. 2, pp. 66-68, marchapril 2008.

[9] A. Davison, I. Reid, N. Molton, and O. Stasse, "Monoslam: Real-time single camera slam," Pattern Analysis and Machine Intelligence, IEEE Transactions on, vol. 29, no. 6, pp. 1052-1067, june 2007.

[10] G. Klein and D. Murray, "Parallel tracking and mapping for small ar workspaces," in Mixed and Augmented Reality, 2007. ISMAR 2007. 6th IEEE and ACM International Symposium on, nov. 2007, pp. 225-234.

[11] E. Foxlin, "Pedestrian tracking with shoe-mounted inertial sensors," Computer Graphics and Applications, IEEE, vol. 25, no. 6, pp. 3846, nov.-dec. 2005.

[12] P. Robertson, M. Angermann, and B. Krach, "Simultaneous localization and mapping for pedestrians using only foot-mounted inertial sensors," in Proceedings of the 11th international conference on Ubiquitous computing (Ubicomp), 2009.

[13] M. Fallon, H. Johannsson, J. Brookshire, S. Teller, and J. Leonard, "Sensor fusion for flexible human-portable building-scale mapping," in Intelligent Robots and Systems (IROS), 2012 IEEE/RSJ International Conference on, oct. 2012, pp. 4405-4412.
[14] J. Haverinen and A. Kemppainen, "A global self-localization technique utilizing local anomalies of the ambient magnetic field," in Robotics and Automation, 2009. ICRA '09. IEEE International Conference on, may 2009.

[15] E. Le Grand and S. Thrun, "3-axis magnetic field mapping and fusion for indoor localization," in Multisensor Fusion and Integration for Intelligent Systems (MFI), 2012 IEEE Conference on, sept. 2012.

[16] B. Gozick, K. Subbu, R. Dantu, and T. Maeshiro, "Magnetic maps for indoor navigation," Instrumentation and Measurement, IEEE Transactions on, vol. 60, no. 12, pp. 3883 -3891, dec. 2011.

[17] T. Riehle, S. Anderson, P. Lichter, J. Condon, S. Sheikh, and D. Hedin, "Indoor waypoint navigation via magnetic anomalies," in Engineering in Medicine and Biology Society,EMBC, 2011 Annual International Conference of the IEEE, sept 2011.

[18] F. Goldenberg, "Geomagnetic navigation beyond the magnetic compass," in IEEE/ION Position Location And Navigation Symposium (PLANS), 2006, april 2006.

[19] J. Moore and R. Tedrake, "Magnetic localization for perching UAVs on powerlines," in Intelligent Robots and Systems (IROS), 2011 IEEE/RSJ International Conference on, sept. 2011.

[20] S.-E. Kim, Y. Kim, J. Yoon, and E. S. Kim, "Indoor positioning system using geomagnetic anomalies for smartphones," in Indoor Positioning and Indoor Navigation (IPIN), 2012 International Conference on, 2012, pp. 1-5.

[21] Y. Sakagami, R. Watanabe, C. Aoyama, S. Matsunaga, N. Higaki, and K. Fujimura, "The intelligent ASIMO: System overview and integration," in Intelligent Robots and Systems, 2002. IEEE/RSJ International Conference on, vol. 3. IEEE, 2002, pp. 2478-2483.

[22] K. Kaneko, K. Harada, F. Kanehiro, G. Miyamori, and K. Akachi, "Humanoid robot HRP-3," in Intelligent Robots and Systems, 2008. IROS 2008. IEEE/RSJ International Conference on. IEEE, 2008, pp. 2471-2478.

[23] K. Kaneko, F. Kanehiro, M. Morisawa, K. Akachi, G. Miyamori, A. Hayashi, and N. Kanehira, "Humanoid robot hrp-4-humanoid robotics platform with lightweight and slim body," in Intelligent Robots and Systems (IROS), 2011 IEEE/RSJ International Conference on. IEEE, 2011, pp. 4400-4407.

[24] A. Shkolnik, M. Levashov, I. R. Manchester, and R. Tedrake, "Bounding on rough terrain with the littledog robot," The International Journal of Robotics Research, vol. 30, no. 2, pp. 192-215, 2011.

[25] M. Raibert, K. Blankespoor, G. Nelson, R. Playter, et al., "Bigdog, the rough-terrain quadruped robot," in Proceedings of the 17th World Congress, 2008, pp. 10823-10825.

[26] J. Chestnutt, M. Lau, G. Cheung, J. Kuffner, J. Hodgins, and T. Kanade, "Footstep planning for the Honda ASIMO humanoid," in Robotics and Automation, 2005. ICRA 2005. Proceedings of the 2005 IEEE International Conference on. IEEE, 2005, pp. 629-634.

[27] N. Kohl and P. Stone, "Policy gradient reinforcement learning for fast quadrupedal locomotion," in Robotics and Automation, 2004. Proceedings. ICRA'04. 2004 IEEE International Conference on, vol. 3. IEEE, 2004, pp. 2619-2624.

[28] H. Kitano, M. Asada, Y. Kuniyoshi, I. Noda, and E. Osawa, "Robocup: The robot world cup initiative," in Proceedings of the first international conference on Autonomous agents. ACM, 1997, pp. 340-347.

[29] M. Sanjeev Arulampalam, S. Maskell, N. Gordon, and T. Clapp, "A tutorial on particle filters for online nonlinear/non-gaussian bayesian tracking," Signal Processing, IEEE Transactions on, vol. 50, no. 2, pp. 174-188, 2002.

[30] M. Angermann, P. Robertson, T. Kemptner, and M. Khider, "A high precision reference data set for pedestrian navigation using footmounted inertial sensors," in Indoor Positioning and Indoor Navigation (IPIN), 2010 International Conference on, sept. 2010.

[31] F. Zampella, M. Khider, P. Robertson, and A. Jimenez, "Unscented kalman filter and magnetic angular rate update (MARU) for an improved pedestrian dead-reckoning," in IEEE/ION Position Location And Navigation Symposium (PLANS), 2012, april 2012.

[32] M. Quigley, K. Conley, B. P. Gerkey, J. Faust, T. Foote, J. Leibs, R. Wheeler, and A. Y. Ng, "ROS: an open-source robot operating system," in ICRA Workshop on Open Source Software, Kobe, Japan, 2009.

[33] J. Hunter, "Matplotlib: A 2D graphics environment," Computing in Science Engineering, vol. 9, no. 3, pp. 90-95, may-june 2009. 